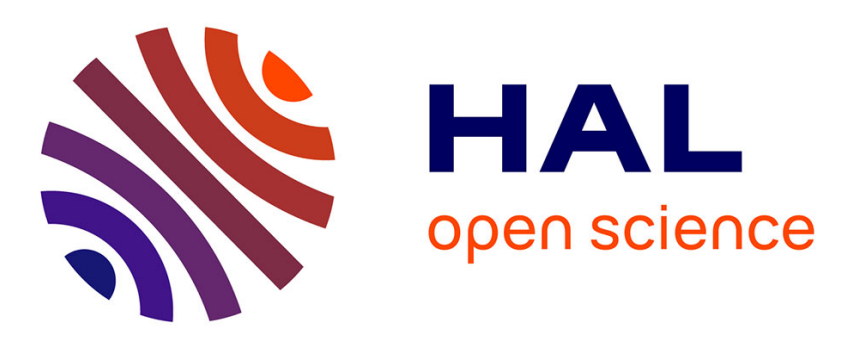

\title{
Ab Initio Study of Helicity-Dependent Light-Induced Demagnetization: From the Optical Regime to the Extreme Ultraviolet Regime
}

Philippe Scheid, Sangeeta Sharma, Grégory Malinowski, Stéphane Mangin, Sébastien Lebègue

\section{To cite this version:}

Philippe Scheid, Sangeeta Sharma, Grégory Malinowski, Stéphane Mangin, Sébastien Lebègue. Ab Initio Study of Helicity-Dependent Light-Induced Demagnetization: From the Optical Regime to the Extreme Ultraviolet Regime. ACS Nano, 2021, 21 (5), pp.1943-1947. 10.1021/acs.nanolett.0c04166 . hal-03433368

\section{HAL Id: hal-03433368 \\ https://hal.univ-lorraine.fr/hal-03433368}

Submitted on 18 Nov 2021

HAL is a multi-disciplinary open access archive for the deposit and dissemination of scientific research documents, whether they are published or not. The documents may come from teaching and research institutions in France or abroad, or from public or private research centers.
L'archive ouverte pluridisciplinaire HAL, est destinée au dépôt et à la diffusion de documents scientifiques de niveau recherche, publiés ou non, émanant des établissements d'enseignement et de recherche français ou étrangers, des laboratoires publics ou privés. 


\title{
$A b$ Initio Study of Helicity-Dependent Light-Induced Demagnetization: From the Optical Regime to the Extreme Ultraviolet Regime
}

\author{
Philippe Scheid,* Sangeeta Sharma, Gregory Malinowski, Stéphane Mangin, and Sébastien Lebègue
}

Cite This: Nano Lett. 2021, 21, 1943-1947

Read Online

ACCESS | Lلll Metrics \& More 回 Article Recommendations

ABSTRACT: We use $a b$ initio real-time time-dependent density functional theory to investigate the effect of optical and extreme ultraviolet (XUV) circularly polarized femtosecond pulses on the magnetization dynamics of ferromagnetic materials. We demonstrate that the light induces a helicity-dependent reduction of the magnitude of the magnetization. In the XUV regime, where the $3 \mathrm{p}$ semicore states are involved, a larger helicity dependence persisting even after the passage of light is exhibited. Finally, we were able to separate the part of the helicity-dependent dynamics due to the absorption from the part due to the inverse Faraday effect. Doing so, we show that

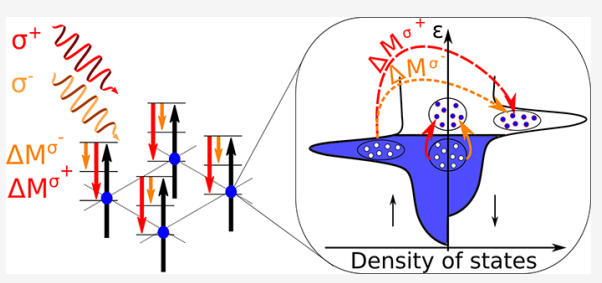
the former has, overall, a greater impact on the magnetization than the latter, especially after the pulse and in the XUV regime. This work hints at the yet experimentally unexplored territory of the XUV light-induced helicity-dependent dynamics, which, according to our prediction, could magnify the helicity-dependent dynamics already exhibited in the optical regime.

KEYWORDS: ab initio calculations, time-dependent density functional theory, ultrafast phenomena, magnetization, helicity-dependent dynamics

$\mathrm{T}$ o face the continuing demand for large-density and energy-efficient data storage devices, the possibilities of manipulating the magnetization without relying on the generation of a magnetic field are widely being investigated. ${ }^{1}$ One of the most promising methods is the use of femtosecond light pulses. Triggered by Beaurepaire et al., this field of study gained a pronounced interest with the discovery of Kimel et $\mathrm{al}^{3}$ that the light-induced magnetization dynamics of an oxide can be helicity dependent. An even more dramatic phenomenon, named the all-optical helicity-dependent switching (AO-HDS), was evidenced in many different types of magnetic thin films the following years. ${ }^{4-7}$ AO-HDS allows for the control of the magnetization state by solely relying on circularly left $\left(\sigma^{+}\right)$or right $\left(\sigma^{-}\right)$polarized light pulses of a few tens of femtoseconds and, as a consequence, is a prime candidate for the disruption of data storage technologies. While the origin of the AO-HDS is still debated, ${ }^{8,9}$ it is assumed that the dominating contribution comes from the inverse Faraday effect (IFE), originally predicted by Pitaevskii $^{10}$ in a nondissipative and nonmagnetic medium. He found that circularly polarized light induces a magnetization proportional to the intensity of the light source at any time, and as a consequence, its contribution to the magnetization dynamics vanishes with the intensity of the light. This effect was recently revisited ${ }^{11,12}$ and evaluated in different absorptive compounds ${ }^{13}$ using $a b$ initio means. Another effect, occurring simultaneously to the IFE in dissipative materials, is the magnetization induced during light absorption ${ }^{14}$ (MILA), whose magnitude is proportional to the fluence of the light. Consequently, and very differently from the IFE, its impact on the magnetization persists even after the presence of the light pulse.

Recently, real-time time-dependent density functional theory (RT-TDDFT) has been successfully applied to describe the linearly polarized laser-induced demagnetization, ${ }^{16}$ and predicted a new phenomenon occurring during the early dynamics based on optical intersite spin transfer, ${ }^{17-19}$ which has later been experimentally confirmed. ${ }^{20-22}$ In the present work, we investigate the dynamics of the magnetization density under the influence of circularly polarized pump pulses using RT-TDDFT. The main aim of the work is to study the dependence of spin dynamics on the helicity of the pump pulse in transition metals (TMs) fcc Ni, fcc Co, and bcc Fe. To this end, we investigate the effect of left and right circularly polarized light of two different kinds, (a) optical pulses and (b) XUV pulses, that resonate with the $3 \mathrm{p}$ states of $\mathrm{Fe}, \mathrm{Co}$, and $\mathrm{Ni}$. We find that, in both cases, the induced spin dynamics is helicity dependent and that this helicity dependence is larger

Received: October 18, 2020

Revised: February 13, 2021

Published: February 19, 2021 
when using a XUV light. The origin of this helicity dependence is closely assessed by examining the spin-resolved timedependent density of states. Then, we separate the contribution of the IFE and the MILA in the helicitydependent part of the magnetization dynamics and show that the latter has a greater impact, especially in the XUV regime and when the light pulse vanishes.

The calculations are performed in two steps: first the ground state of the system is calculated using density functional theory. ${ }^{23,24}$ RT-TDDFT is then used to time propagate this ground state under the influence of a pump pulse which is treated as an external vector potential. These calculations are performed with the noncollinear full potential linearized augmented-plane-wave method, ${ }^{25}$ including spin-orbit coupling, as implemented in the ELK code (http://elk. sourceforge.net/). The Brillouin zone is sampled with a $20 \times$ $20 \times 20$ mesh for each system, and states up to $90 \mathrm{eV}$ above the Fermi energy are included. Within RT-TDDFT, ${ }^{26}$ the dynamics of the Kohn-Sham spinors is evaluated using

$$
\begin{aligned}
\frac{\partial \phi_{n \mathbf{k}}(\mathbf{r}, t)}{\partial t}= & \left(-\frac{1}{2} \nabla^{2}-\frac{i}{c} \mathbf{A}_{\mathrm{ext}}(t) \cdot \nabla+v_{\mathrm{s}}(\mathbf{r}, t)+\frac{1}{2 c} \boldsymbol{\sigma} \cdot \mathbf{B}_{\mathrm{xc}}(\mathbf{r}, t)\right. \\
& \left.-\frac{1}{4 c^{2}} \boldsymbol{\sigma}\left(\nabla v_{\mathrm{s}}(\mathbf{r}, t) \times i \nabla\right)\right) \phi_{n \mathbf{k}}(\mathbf{r}, t)
\end{aligned}
$$

In this equation $\phi_{n \mathbf{k}}(\mathbf{r}, t)=\langle\mathbf{r} \mid n \mathbf{k}(t)\rangle$ is a two-component spinor valued Bloch orbital indexed by $\mathbf{k}$, a vector of the first Brillouin zone and $n$ the band index; $\mathbf{A}_{\mathrm{ext}}$ is the vector potential of the light; $\boldsymbol{\sigma}$ are the Pauli matrices; $\mathbf{B}_{\mathrm{xc}}(\mathbf{r}, t)$ is the exchange correlation magnetic field; and $v_{s}(\mathbf{r}, t)$ is the effective KohnSham potential. The latter is the sum of the external potential, $v_{\text {ext }}(\mathbf{r}, t)$, the Hartree potential, $v_{\mathrm{H}}(\mathbf{r}, t)$, and the exchange correlation potential, $v_{x c}(\mathbf{r}, t)$. To compute the exchange correlation potential, we use the noncollinear ${ }^{27}$ adiabatic local spin density approximation. The last term of the right-hand side of eq 1 is the spin-orbit coupling. As shown in previous works, ${ }^{13,14}$ its presence is required in order for the magnetization to be affected by the light. While, as discussed by Mondal et al., ${ }^{28,29}$ it is not the only relativistic contribution appearing up to the second-order expansion of the Dirac Hamiltonian, its contribution to the dynamics is far greater than the others, ${ }^{29}$ which are therefore safely left out here. The magnetization is treated as a fully unconstrained vector field, and the magnetic moment is evaluated using

$$
\mathbf{M}(t)=\mu_{\mathrm{B}} \sum_{n} \frac{1}{\Omega_{\mathrm{BZ}}} \int_{\mathrm{BZ}} \mathrm{d} \mathbf{k}\langle n \mathbf{k}(t)|\hat{\boldsymbol{\sigma}}| n \mathbf{k}(t)\rangle f_{n \mathbf{k}}
$$

where $f_{n \mathbf{k}}$ is the occupation of the state $|n \mathbf{k}\rangle$ defined by the initial state of the Kohn-Sham system; $\mu_{\mathrm{B}}$ is the Bohr magneton; and $\Omega_{\mathrm{BZ}}$ is the volume of the first Brillouin zone.

The last term in eq 1 is the spin-orbit coupling. Without it, $\boldsymbol{\sigma}$ would commute with the Hamiltonian, and the magnetization would remain constant during the dynamics, as shown in ref 15 . The time propagation is done using the algorithm detailed in ref 17 with a time step of $2.4 \mathrm{~s}$.

The dynamics of the charge and spin density of bcc Fe, fcc $\mathrm{Co}$, and $\mathrm{fcc} \mathrm{Ni}$ are evaluated under the action of circularly polarized laser pulses having a Gaussian intensity profile of $\mathrm{fwhm}=25 \mathrm{fs}$ and an incident fluence of $13.11 \mathrm{~mJ} / \mathrm{cm}^{2}$. Simulations were performed with an optical pulse of frequency of $1.55 \mathrm{eV}$ and XUV pulses resonating with the lowest-lying $3 \mathrm{p}$ states, respectively, located at 55, 60, and $65 \mathrm{eV}$ below the Fermi energy in bcc $\mathrm{Fe}$, fcc $\mathrm{Co}$, and fcc Ni. The pump pulse propagates along the direction of the average magnetic moment, which we define as the $z$-axis.

Both Figure 1(a) and (b) show that, as for linearly polarized pump pulses, ${ }^{15}$ circularly polarized pump pulses also lead to
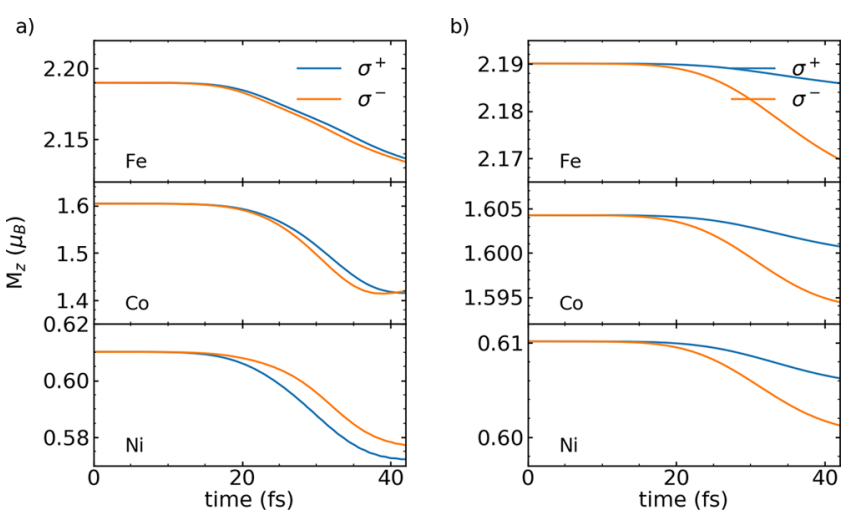

Figure 1. RT-TDDFT dynamics of the $z$ component of the magnetic moment, $M_{z}$ calculated for bcc $\mathrm{Fe}$, fcc $\mathrm{Co}$, and fcc Ni performed at $1.55 \mathrm{eV}$ (a) and in the XUV range (b).

spin-orbit coupling mediated ${ }^{14,30}$ demagnetization of TMs. We find that for a fixed incident fluence the demagnetization is more pronounced when using an optical pump pulse, rather than a XUV one. The helicity-dependent magnetization dynamics can also be clearly seen in Figure 1; for an optical pulse, with the exception of $\mathrm{Ni}$, demagnetization induced by $\sigma^{-}$ is larger than $\sigma^{+}$. This particular behavior of $\mathrm{Ni}$ was also noted in ref 14. For XUV pulses, $\sigma^{-}$always leads to a larger demagnetization than its counterpart for all of the studied materials. Also, the difference between the demagnetization from two helicities is much more pronounced for XUV pulses as compared to optical pulses.

These results pose two interesting questions: (i) Why larger demagnetization is produced by optical as compared to XUV pulses? (ii) Why the helicity dependence is more pronounced for XUV pulses than for optical pulses? In order to understand this, we now proceed to the computation of the transient spinresolved occupied density of states (oDOS) defined as ${ }^{18}$

$$
\operatorname{oDOS}^{\sigma}(\varepsilon, t)=\frac{1}{\Omega_{\mathrm{BZ}}} \sum_{n} \int_{\Omega_{\mathrm{BZ}}} \mathrm{d} \mathbf{k}\left[f_{n \mathbf{k}}(t) \cdot\langle n \mathbf{k} \mid \sigma\rangle\langle\sigma \mid n \mathbf{k}\rangle \delta\left(\varepsilon_{n \mathbf{k}}-\varepsilon\right)\right]
$$

where $\varepsilon$ is the energy; $t$ is the time; $\varepsilon_{n \mathbf{k}}$ is the eigenvalue associated with the Bloch eigenstate $|n \mathbf{k}\rangle$; and $\sigma$ is indexing the spin state, which for a collinear system would be $\uparrow$ or $\downarrow$. Finally $f_{n \mathbf{k}}(t)=\sum_{m} f_{m \mathbf{k}}|\langle n \mathbf{k} \mid m \mathbf{k}(t)\rangle|^{2}$ is the time-dependent occupation factor of the state $|n \mathbf{k}\rangle$.

Figure $2(\mathrm{a}-\mathrm{d})$ show the changes in oDOS for bcc Fe; i.e., $\operatorname{oDOS}^{\sigma}(t=32 \mathrm{fs})-\operatorname{oDOS}^{\sigma}(t=0 \mathrm{fs})$, for a $\sigma^{+}$and a $\sigma^{-}$ polarization of an optical and a XUV pulse. In both cases, this quantity decreases below the ground-state Fermi level $(0 \mathrm{eV})$ and increases above it; i.e., electrons are getting excited and start occupying empty states. Excitations at energies well below $1.55 \mathrm{eV}$ show the presence of nonlinear processes. Comparing Figure 2(a) and (b) with (d) and (e), question (i) is immediately answered: the magnitude of change in the oDOS is almost 25 times larger for both helicities of the optical light, as opposed to the XUV one; i.e., the optical pulse is more absorbed than the XUV pulse. Consequently, and as explained in ref 14 , this leads to a larger number of excited electrons 

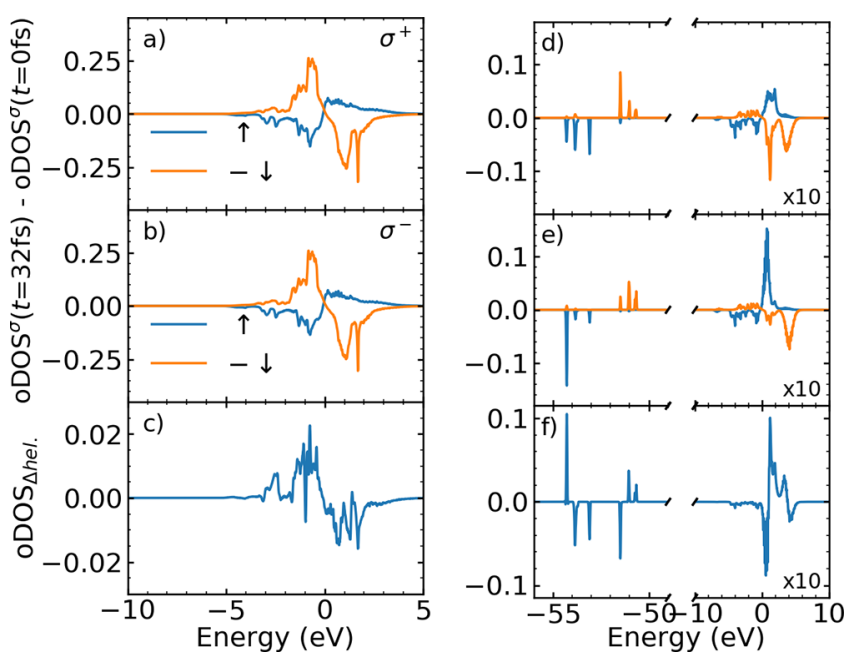

Figure 2. $(\mathrm{a}-\mathrm{e})$ Variation of the spin- and occupation-resolved density of states, $\operatorname{oDOS}^{\sigma}(t=32 \mathrm{fs})-\mathrm{oDOS}^{\sigma}(t=0 \mathrm{fs})$, in bcc Fe, induced by a $\sigma^{+}(\mathrm{a})$ and (d) and a $\sigma^{-}(\mathrm{b})$ and (e) polarization of the light induced after $32 \mathrm{fs}$ using an optical ( $\mathrm{a}$ and $\mathrm{b}$ ) and a XUV light (d and e). To improve readability, the $\downarrow$ curves are multiplied by -1 . (c, f) Difference of the total density of states induced by a $\sigma^{+}$and a $\sigma^{-}$ polarized light, $\mathrm{oDOS}_{\Delta \text { hel. }}=\mathrm{oDOS}_{\sigma^{+}}(t=32 \mathrm{fs})-\mathrm{oDOS}_{\sigma}^{-}(t=32 \mathrm{fs})$, after $32 \mathrm{fs}$ computed at $1.55 \mathrm{eV}$ (in the XUV range). The right side of (d), (e), and (f) is magnified by a factor of 10 .

going from the majority states to the minority states, which are preponderant above the Fermi energy and hence lead to a larger demagnetization by the optical pulse.

In order to answer question (ii) let us first recall that dipolar coupling imposes that transitions induced by a $\sigma^{ \pm}$-polarized light satisfy $\Delta j= \pm 1$ for $\sigma^{ \pm}$and $\Delta m_{j}= \pm 1$, where $j$ is the total angular momentum. However, in solids, valence electrons rearrange themselves to form bonds, and $j$ is not a good quantum number anymore. Nevertheless, 3d states, more localized, and especially $3 p$ semicore states do not hybridize as much, and the aforementioned helicity-dependent selection rules may, at least partially, apply. To confirm this, in Figure 2 (c) and (f) we look at the change in spin-integrated DOS produced by the two different helicities after 32 fs: $\mathrm{oDOS}_{\Delta \text { hel. }}=\mathrm{oDOS}_{\sigma^{+}}(t=32 \mathrm{fs})-\mathrm{oDOS}_{\sigma^{-}}(t=32 \mathrm{fs})$. In the optical range, the helicity-dependent effects that were the strongest were the DOS peaks (see the total DOS in ref 31). Indeed, these peaks reveal the presence of $3 \mathrm{~d}$ states, for which the aforementioned helicity-dependent selection rules apply more. This difference is 5 times more pronounced in the XUV range involving the $3 \mathrm{p}$ semicore states, whose negligible dispersion is evidenced by the sharpness of the peaks they exhibit in the DOS. This answers question (ii).

To further highlight the different HD dynamics induced by the two different kinds of pulses, we decompose $M_{z}^{\sigma^{ \pm}}(t)$ in a helicity-independent and a helicity-dependent part as

$$
M_{z}^{\sigma^{ \pm}}(t)=M_{\mathrm{HI}}(t) \pm M_{\mathrm{HD}}(t)
$$

where $M_{z}^{\sigma^{ \pm}}(t)$ is the $z$ component of the time-dependent magnetization, for which dynamics is triggered by a circularly left $\left(\sigma^{+}\right)$or right $\left(\sigma^{-}\right)$polarized light. The helicity-independent part writes as $M_{\mathrm{HI}}(t)=\frac{1}{2}\left(M_{z}^{\sigma^{+}}(t)+M_{z}^{\sigma^{-}}(t)\right)$ and the helicitydependent part as $M_{\mathrm{HD}}(t)=\frac{1}{2}\left(M_{z}^{\sigma^{+}}(t)-M_{z}^{\sigma^{-}}(t)\right)$. Using this framework, we define

$$
\zeta(t)=\frac{\left|M_{\mathrm{HD}}(t)\right|}{M_{\mathrm{HI}}(0)-M_{\mathrm{HI}}(t)}
$$

representing the relative magnitude of the $\mathrm{HD}$ effect over the $\mathrm{HI}$ one (both $M_{\mathrm{HD}}(t)$ and $M_{\mathrm{HI}}(t)$ have been convoluted with a Gaussian having a standard deviation of $2 \mathrm{fs}$ ). Figure 3(b) and
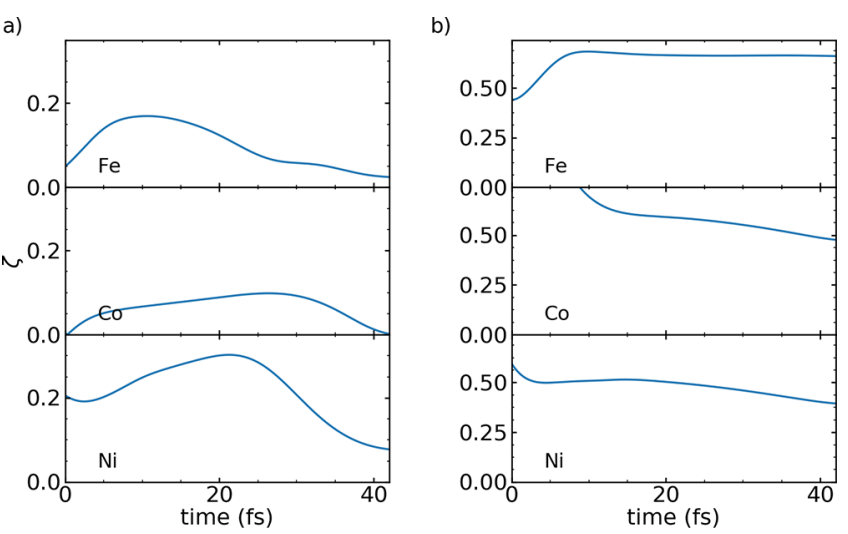

Figure 3. Magnitude of the helicity-dependent dynamics over the helicity-independent one, $\zeta$, at $1.55 \mathrm{eV}$ (a) and in the XUV range (b).

(d) are plots of $\zeta$ as a function of time for both types of pulses. They evidence that HD effects are more than twice as large in the XUV range than at $1.55 \mathrm{eV}$. Moreover, while in the optical regime $\zeta$ tends to be attenuated by the complex dynamics of the interacting electronic density, the light-induced helicity dependence is maintained over a longer course of time in the XUV regime.

In the past, there has been discussion about the factors that lead to HD dynamics. The two main candidates have been identified to be the IFE and the MILA. This past analysis has been done assuming that the magnetic response of the system is dominated by linear order in perturbation. Even though, as mentioned above, our work also includes a nonlinear effect, in order to comment on the relative importance of these contributions, we perform the following analysis: according to Battiato et al. ${ }^{11,12} M_{\mathrm{HD}}(t)$ is composed of a transient signal, oscillating parts, the absorptive contribution which leads to MILA, ${ }^{14}$ and the zeroth harmonic which leads to the IFE. ${ }^{10-13}$ To remove the oscillatory and transient contributions which together time average to 0 , we perform a convolution of $M_{\mathrm{HD}}$ with a Gaussian having a root-mean-square of $2 \mathrm{fs}$. This allows us to decompose the $\mathrm{HD}$ part of the magnetization dynamics into

$$
M_{\mathrm{HD}}(t) \approx M_{\mathrm{I}}(t)+M_{\mathrm{F}}(t)=\gamma_{\mathrm{I}} I(t)+\gamma_{\mathrm{F}} \int_{0}^{t} \mathrm{~d} t^{\prime} I\left(t^{\prime}\right)
$$

where $I(t)$ is the intensity of the light; and $\gamma_{\mathrm{I}}$ and $\gamma_{\mathrm{F}}$ are, respectively, characterizing the magnitude of the $\mathrm{HD}$ effect proportional to the intensity, such as the zeroth harmonic generation, and to the fluence, such as the absorption. As presented by Battiato et al., ${ }^{11}$ and as seen in the results discussed below, the magnitude of both $\gamma_{\mathrm{I}}$ and $\gamma_{\mathrm{F}}$ depends on the distribution of frequencies present in the laser pulse. In this framework, the contribution of both effects in $M_{\mathrm{HD}}$ is uniquely defined by 


$$
\gamma_{\mathrm{I}}=\frac{\frac{\partial}{\partial t}\left(\frac{M_{\mathrm{HD}}(t)}{\int_{0}^{t} \mathrm{~d} t^{\prime} I\left(t^{\prime}\right)}\right)}{\frac{\partial}{\partial t}\left(\frac{I(t)}{\int_{0}^{t} \mathrm{~d} t^{\prime} I\left(t^{\prime}\right)}\right)}
$$

and

$$
\gamma_{\mathrm{F}}=\frac{\frac{\partial}{\partial t}\left(\frac{M_{\mathrm{HD}}(t)}{I(t)}\right)}{1-\frac{\frac{\partial}{\partial t} I(t)}{I^{2}(t)} \int_{0}^{t} \mathrm{~d} t^{\prime} I\left(t^{\prime}\right)}
$$

The individual contributions of $M_{\mathrm{I}}(t)$ and $M_{\mathrm{F}}(t)$ to the total magnetization density are shown in Figure 4 for bcc Fe, fcc Co, and fcc Ni.

In the case of the optical pump pulse (Figure 4(a)), the magnitudes of both effects (MILA and IFE) are similar during
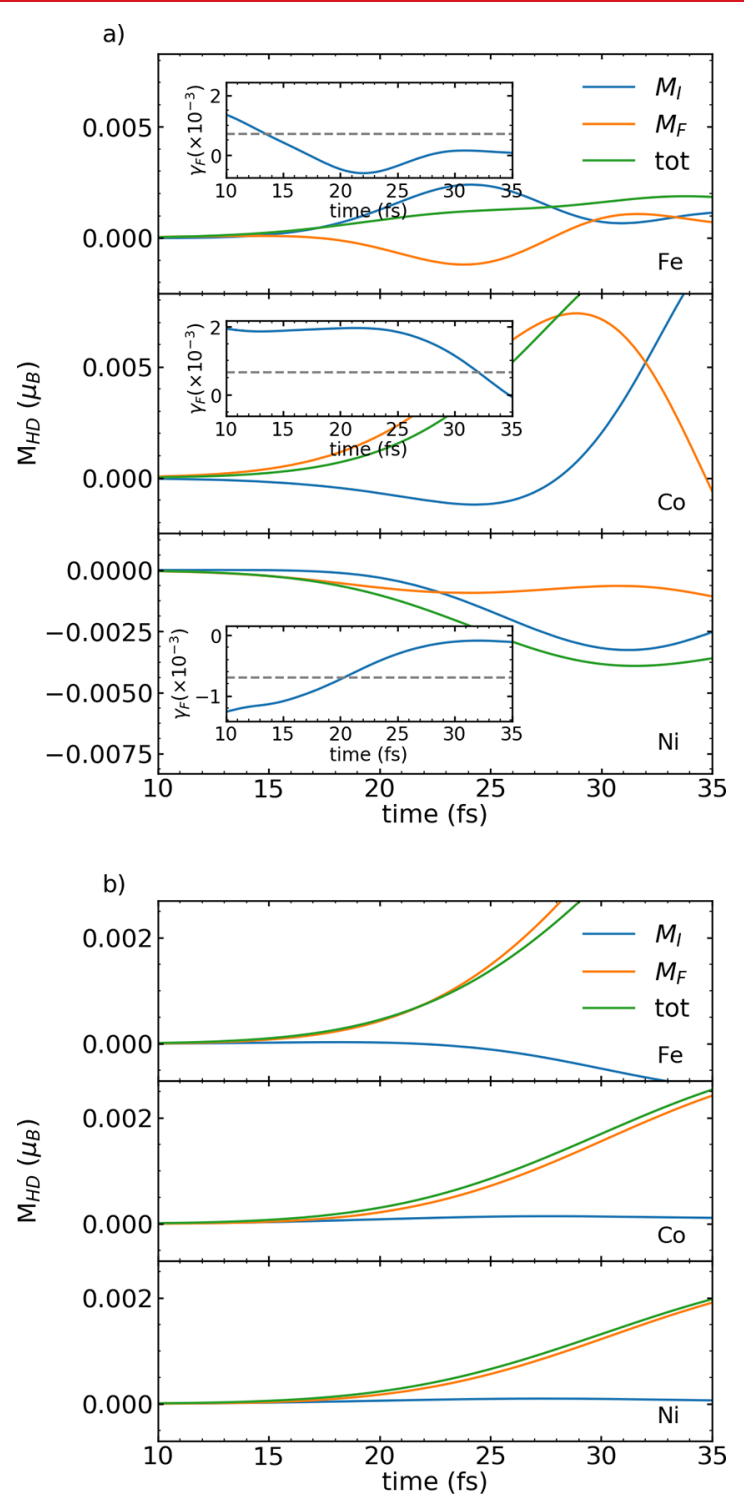

Figure 4. Zeroth harmonic $\left(M_{\mathrm{I}}\right)$ and absorption $\left(M_{\mathrm{F}}\right)$ part of the helicity-dependent dynamics calculated with eq 6 , where $M_{\mathrm{HD}}$ is induced by a light of $1.55 \mathrm{eV}$ (a) and in the XUV regime (b). The dashed lines in (a) represent the contribution to the dynamics of the angular momentum absorption effect, calculated in ref 14 . the pulse. The part of the HD dynamics due to the absorption, $M_{\mathrm{F}}$, can be compared to some earlier work ${ }^{14}$ using standard density functional theory, and as seen in the inset of Figure 4(a), we find that at the early stages of the dynamics, where the linear regime is dominant, both the signs and the magnitudes are in agreement.

In the XUV range (Figure 4(b)), the dynamics is dominated by the absorption (MILA), i.e., $M_{\mathrm{HD}} \approx M_{\mathrm{F}}$. Consequently, the helicity-dependent magnetization induced by the XUV light will mostly persist even after its intensity has decreased and a large $\zeta$ is obtained at all times, in comparison with the optical excitation (see Figure 3).

In conclusion, using real-time time-dependent density functional theory, we evidenced the presence of a helicitydependent magnetization dynamics in fcc Ni, fcc Co, and bcc $\mathrm{Fe}$ characterized by a difference in the reduction of the magnetic moment carried by the atoms. We found that this helicity dependence is particularly strong when exciting the more localized $3 p$ states. Indeed, by closely examining the time-dependent density of states, we found that the helicity dependence of the dynamics is enhanced for localized states. Then, we disentangled the role of the two main first-order effects which may be held responsible for the helicity dependence, i.e., the inverse Faraday effect and the angular momentum absorption effect. We found that the latter has a far greater impact than the former on the overall dynamics, especially in the XUV range and after the light is gone.

These results also have strong experimental implications and may be responsible for the helicity-dependent demagnetization evidenced by Tsema et al. ${ }^{32}$ and Quessab et al. ${ }^{33}$ Furthermore, the dynamics reported here may also play a role in the AO$\operatorname{HDS}^{6,7}$ and the helicity-dependent domain wall motion. ${ }^{34}$ Finally, our results are a call for experimental exploration, as they suggest a large enhancement of the efficiency of all of the aforementioned phenomena by going out of the optical range and, in doing so, involving localized core and semicore states.

\section{AUTHOR INFORMATION}

\section{Corresponding Author}

Philippe Scheid - Université de Lorraine, LPCT, CNRS, UMR 7019, 54506 Cedex Vandoeuvre-lès-Nancy, France; Université de Lorraine, IJL, CNRS, UMR 7198, 54000 Cedex Nancy, France; (이이.org/0000-0002-4440-4539; Email: philippe.scheid@univ-lorraine.fr

\section{Authors}

Sangeeta Sharma - Max-Born-Institute for Non-linear Optics and Short Pulse Spectroscopy, 12489 Berlin, Germany

Gregory Malinowski - Université de Lorraine, IJL, CNRS, UMR 7198, 54000 Cedex Nancy, France

Stéphane Mangin - Université de Lorraine, IJL, CNRS, UMR 7198, 54000 Cedex Nancy, France; 이이이.org/00000001-6046-0437

Sébastien Lebègue - Université de Lorraine, LPCT, CNRS, UMR 7019, 54506 Cedex Vandoeuvre-lès-Nancy, France

Complete contact information is available at:

https://pubs.acs.org/10.1021/acs.nanolett.0c04166

\section{Notes}

The authors declare no competing financial interest. 


\section{ACKNOWLEDGMENTS}

The authors are grateful to J. K. Dewhurst, P. Elliott, and D. Malterre for the many insightful and stimulating discussions. P. Scheid was supported by the French PIA project Lorraine Université d'Excellence, reference ANR-15-IDEX-04-LUE. S. Sharma would like to acknowledge DFG for funding through SPP QUTIF. G. Malinowski, S. Mangin, and S. Lebègue acknowledge financial support from the ANR through the UFO project (ANR-20-CE09-0013).

\section{REFERENCES}

(1) Sander, D.; et al. The 2017 Magnetism Roadmap. J. Phys. D: Appl. Phys. 2017, 50, 363001.

(2) Beaurepaire, E.; Merle, J. C.; Daunois, A.; Bigot, J. Y. Ultrafast spin dynamics in ferromagnetic nickel. Phys. Rev. Lett. 1996, 76, $4250-4253$

(3) Kimel, A. V.; Kirilyuk, A.; Usachev, P. A.; Pisarev, R. V.; Balbashov, A. M.; Rasing, T. Ultrafast non-thermal control of magnetization by instantaneous photomagnetic pulses. Nature 2005, $435,655-657$.

(4) Stanciu, C. D.; Hansteen, F.; Kimel, A. V.; Kirilyuk, A.; Tsukamoto, A.; Itoh, A.; Rasing, T. All-optical magnetic recording with circularly polarized light. Phys. Rev. Lett. 2007, 99, 47601.

(5) Alebrand, S.; Gottwald, M.; Hehn, M.; Steil, D.; Cinchetti, M.; Lacour, D.; Fullerton, E. E.; Aeschlimann, M.; Mangin, S. Lightinduced magnetization reversal of high-anisotropy $\mathrm{TbCo}$ alloy films. Appl. Phys. Lett. 2012, 101, 162408.

(6) Mangin, S.; Gottwald, M.; Lambert, C. H.; Steil, D.; Uhlír, V.; Pang, L.; Hehn, M.; Alebrand, S.; Cinchetti, M.; Malinowski, G.; Fainman, Y.; Aeschlimann, M.; Fullerton, E. E. Engineered materials for all-optical helicity-dependent magnetic switching. Nat. Mater. 2014, 13, 286-292.

(7) Lambert, C. H.; Mangin, S.; Varaprasad, B. S. S.; Takahashi, Y. K.; Hehn, M.; Cinchetti, M.; Malinowski, G.; Hono, K.; Fainman, Y.; Aeschlimann, M.; Fullerton, E. E. All-optical control of ferromagnetic thin films and nanostructures. Science 2014, 345, 1337-1340.

(8) Gorchon, J.; Yang, Y.; Bokor, J. Model for multishot all-thermal all-optical switching in ferromagnets. Phys. Rev. B: Condens. Matter Mater. Phys. 2016, 94, 020409.

(9) Ellis, M. O.; Fullerton, E. E.; Chantrell, R. W. All-optical switching in granular ferromagnets caused by magnetic circular dichroism. Sci. Rep. 2016, 6, 30522.

(10) Pitaevskii, L. P. Electric Forces in a Transparent Dispersive Medium. J. Exptl. Theoret. Phys. (U.S.S.R.) 1961, 12, 1450.

(11) Battiato, M.; Barbalinardo, G.; Carva, K.; Oppeneer, P. M. Beyond linear response theory for intensive light-matter interactions: Order formalism and ultrafast transient dynamics. Phys. Rev. B: Condens. Matter Mater. Phys. 2012, 85, 045117.

(12) Battiato, M.; Barbalinardo, G.; Oppeneer, P. M. Quantum theory of the inverse Faraday effect. Phys. Rev. B: Condens. Matter Mater. Phys. 2014, 89, 014413.

(13) Berritta, M.; Mondal, R.; Carva, K.; Oppeneer, P. M. Ab Initio Theory of Coherent Laser-Induced Magnetization in Metals. Phys. Rev. Lett. 2016, 117, 137203.

(14) Scheid, P.; Malinowski, G.; Mangin, S.; Lebègue, S. Ab initio theory of magnetization induced by light absorption in ferromagnets. Phys. Rev. B: Condens. Matter Mater. Phys. 2019, 100, 214402.

(15) Krieger, K.; Dewhurst, J. K.; Elliott, P.; Sharma, S.; Gross, E. K. Laser-Induced Demagnetization at Ultrashort Time Scales: Predictions of TDDFT. J. Chem. Theory Comput. 2015, 11, 4870-4874.

(16) Krieger, K.; Elliott, P.; Müller, T.; Singh, N.; Dewhurst, J. K.; Gross, E. K.; Sharma, S. Ultrafast demagnetization in bulk versus thin films: An ab initio study. J. Phys.: Condens. Matter 2017, 29, 224001.

(17) Dewhurst, J. K.; Krieger, K.; Sharma, S.; Gross, E. K. An efficient algorithm for time propagation as applied to linearized augmented plane wave method. Comput. Phys. Commun. 2016, 209, $92-95$.
(18) Elliott, P.; Müller, T.; Dewhurst, J. K.; Sharma, S.; Gross, E. K. Ultrafast laser induced local magnetization dynamics in Heusler compounds. Sci. Rep. 2016, DOI: 10.1038/srep38911.

(19) Dewhurst, J. K.; Shallcross, S.; Gross, E. K.; Sharma, S. Substrate-Controlled Ultrafast Spin Injection and Demagnetization. Phys. Rev. Appl. 2018, 10, 44065.

(20) Siegrist, F.; Gessner, J. A.; Ossiander, M.; Denker, C.; Chang, Y. P.; Schröder, M. C.; Guggenmos, A.; Cui, Y.; Walowski, J.; Martens, U.; Dewhurst, J. K.; Kleineberg, U.; Münzenberg, M.; Sharma, S.; Schultze, M. Light-wave dynamic control of magnetism. Nature 2019, $571,240-244$.

(21) Chen, J.; Bovensiepen, U.; Eschenlohr, A.; Müller, T.; Elliott, P.; Gross, E. K.; Dewhurst, J. K.; Sharma, S. Competing Spin Transfer and Dissipation at $\mathrm{Co} / \mathrm{Cu}$ (001) Interfaces on Femtosecond Timescales. Phys. Rev. Lett. 2019, DOI: 10.1103/PhysRevLett.122.067202.

(22) Hofherr, M.; Hauser, S.; Dewhurst, J. K.; Tengdin, P.; Sakshath, S.; Nembach, H. T.; Weber, S. T.; Shaw, J. M.; Silva, T. J.; Kapteyn, H. C.; Cinchetti, M.; Rethfeld, B.; Murnane, M. M.; Steil, D.; Stadtmuller, B.; Sharma, S.; Aeschlimann, M.; Mathias, S.; et al. Ultrafast optically induced spin transfer in ferromagnetic alloys. Science Advances 2020, 6, 1-8.

(23) Hohenberg, P.; Kohn, W. Inhomogeneous electron gas. Phys. Rev. 1964, 136, B864-B871.

(24) Kohn, W.; Sham, L. J. Self-consistent equations including exchange and correlation effects. Phys. Rev. 1965, 140, A1133-A1138.

(25) Perdew, J. P.; Wang, Y. Accurate and simple analytic representation of the electron-gas correlation energy. Phys. Rev. B: Condens. Matter Mater. Phys. 1992, 45, 13244-13249.

(26) Runge, E.; Gross, E. K. Density-functional theory for timedependent systems. Phys. Rev. Lett. 1984, 52, 997-1000.

(27) Kubler, J; Hock, K -H; Sticht, J; Williams, A R Density functional theory of non-collinear magnetism. J. Phys. F: Met. Phys. 1988, 18, 469-483.

(28) Mondal, R.; Berritta, M.; Paillard, C.; Singh, S.; Dkhil, B.; Oppeneer, P. M.; Bellaiche, L. Relativistic interaction Hamiltonian coupling the angular momentum of light and the electron spin. Phys. Rev. B: Condens. Matter Mater. Phys. 2015, 92, 1-4.

(29) Mondal, R.; Berritta, M.; Carva, K.; Oppeneer, P. M. Ab initio investigation of light-induced relativistic spin-flip effects in magnetooptics. Phys. Rev. B: Condens. Matter Mater. Phys. 2015, 91, 1-9.

(30) Elliott, P.; Singh, N.; Krieger, K.; Gross, E. K. U.; Sharma, S.; Dewhurst, J. K. The microscopic origin of spin-orbit mediated spinflips. J. Magn. Magn. Mater. 2020, 502, 166473.

(31) Willems, F.; Sharma, S.; Korff Schmising, C. V.; Dewhurst, J. K.; Salemi, L.; Schick, D.; Hessing, P.; Strüber, C.; Engel, W. D.; Eisebitt, S. Magneto-Optical Functions at the $3 p$ Resonances of $\mathrm{Fe}$, $\mathrm{Co}$, and Ni: $\mathrm{Ab}$ initio Description and Experiment. Phys. Rev. Lett. 2019, DOI: $10.1103 /$ PhysRevLett.122.217202.

(32) Tsema, Y.; Kichin, G.; Hellwig, O.; Mehta, V.; Kimel, A. V.; Kirilyuk, A.; Rasing, T. Helicity and field dependent magnetization dynamics of ferromagnetic Co/Pt multilayers. Appl. Phys. Lett. 2016, $109,072405$.

(33) Quessab, Y.; Deb, M.; Gorchon, J.; Hehn, M.; Malinowski, G.; Mangin, S. Resolving the role of magnetic circular dichroism in multishot helicity-dependent all-optical switching. Phys. Rev. B: Condens. Matter Mater. Phys. 2019, DOI: 10.1103/PhysRevB.100.024425.

(34) Quessab, Y.; Medapalli, R.; El Hadri, M. S.; Hehn, M.; Malinowski, G.; Fullerton, E. E.; Mangin, S. Helicity-dependent alloptical domain wall motion in ferromagnetic thin films. Phys. Rev. B: Condens. Matter Mater. Phys. 2018, 97, 054419. 